\title{
The Importance of Early Diagnosis and Management of Pediatric Neurogenic Bladder Dysfunction
}

\author{
K Tyler Hobbs' \\ Madison Krischak ${ }^{2}$ \\ Rohit Tejwani' \\ J Todd Purves (1D) \\ John S Wiener (D) \\ Jonathan C Routh (D) \\ 'Division of Urologic Surgery, Duke \\ University Medical Center, Durham, NC, \\ USA; ${ }^{2}$ Duke University School of \\ Medicine, Durham, NC, USA
}

\begin{abstract}
Neurogenic bladder dysfunction is a major source of urologic morbidity in children, especially in those with spina bifida (SB). Complications from progression of bladder dysfunction can include urinary tract infections (UTIs), urinary incontinence, upper tract deterioration, and renal dysfunction or failure. In these children, there has been a recent trend toward proactive rather than expectant management of neurogenic bladder. However, there is a lack of consensus on how to best achieve the three main goals of neurogenic bladder management: 1) preserving kidney function, 2) achieving continence (if desired by the family/individual), and 3) achieving social and functional urologic independence (if appropriate). Hence, our objective was to perform a narrative literature review to evaluate the approaches to diagnosis and management of pediatric neurogenic bladder dysfunction, with special focus on children with SB. The approach strategies vary across a spectrum, with a proactive strategy on one end of the spectrum and an expectant strategy at the other end. The proactive management strategy is characterized by early and frequent labs, imaging, and urodynamic (UDS) evaluation, with early initiation of clean intermittent catheterization (CIC) and proceeding with pharmacotherapy, or surgery if indicated. The expectant management strategy prioritizes surveillance labs and imaging prior to proceeding with invasive assessments and interventions such as UDS or pharmacotherapy. Both treatment strategies are currently utilized and data have historically been inconclusive in demonstrating efficacy of one regimen over the other. We performed a narrative literature evaluating proactive and expectant treatment strategies as they relate to diagnostics and management of Spina Bifida. From the available literature and our practice, a proactive strategy favors greater benefit in preventative management and may decrease risk of renal dysfunction compared with expectant management.
\end{abstract}

Keywords: bladder dysfunction, management, pediatric, neurogenic bladder, spina bifida, diagnosis

\section{Introduction}

Neurogenic bladder dysfunction is a major source of urologic morbidity in children, especially in those with spina bifida (SB). ${ }^{1,2} \mathrm{SB}$ is the most common permanently disabling birth defect in the United States, ${ }^{3}$ affecting 3.5 of every 10,000 live births. ${ }^{4-6}$ Individuals with SB and neurogenic bladder are at risk for chronic kidney disease, urinary tract infections (UTIs), and urinary incontinence. ${ }^{6,7}$ The primary urologic goals of SB management is preservation of lifetime renal function, achieve urinary continence, and maximization of urologic independence as appropriate. ${ }^{7-9}$
Correspondence: Jonathan C Routh Division of Urologic Surgery, Duke University Medical Center, DUMC 383I, Durham, NC, 277I0, USA

Tel +I (919) 684-6994

Fax + I (919) 68I-5507

Email jonathan.routh@duke.edu 
While significant improvements in care of neurogenic bladder and SB have been made in recent decades, the optimal urologic management of SB-related bladder dysfunction is not well defined. ${ }^{10}$ Historically, patients were observed until symptoms or signs of neurogenic bladder or renal damage developed prior to intervention. ${ }^{11}$ This is an expectant approach to management; some may refer to this as a selective or reactive treatment approach. Recently, there has been a trend toward a proactive, or early, approach - to intervene before any renal damage could start to develop. ${ }^{6,12}$ This strategy was initially popularized by Bauer et $\mathrm{al}^{13}$ and is perhaps best exemplified by the ongoing, CDC-funded, Urologic Management to Preserve Initial REnal function (UMPIRE) trial. ${ }^{6}$ The proactive and expectant approaches are at opposite ends of the spectrum of management and some practitioners likely fall somewhere in between, practicing a more hybrid approach. In our practice, we have found that the proactive approach has a greater benefit in preventative management and may decrease risk of renal dysfunction compared with expectant management.

However, there is still uncertainty regarding the best approach for managing pediatric neurogenic bladder and SB-related urologic care, there is a clear opportunity for further optimization, which would presumably lead to protection of renal function, improved urinary continence, reduced UTI rates, improved quality of life, ${ }^{14}$ and decreased need for urologic surgery. ${ }^{6}$ In addition, with inpatient and emergency department healthcare costs for SB-related care estimated at more than $\$ 2$ billion per year in the US alone, investigating optimal management strategies is paramount for the practice of high value care. ${ }^{15}$

Our objective in this narrative review was to review the current literature to provide an overview of proactive and expectant management of pediatric neurogenic bladder dysfunction, with special focus on those with SB. We will focus on diagnostics and treatment with a detailed discussion of each modality in the context of a proactive and expectant strategies. An overview of proactive and expectant management approaches is provided in Table 1.

\section{Diagnostics}

\section{Imaging}

Kidney dysfunction in patients with SB and neurogenic bladder is often silent, necessitating the need for regular surveillance imaging of the upper urinary tract. While imaging is a cornerstone of both proactive and expectant management strategies, it is wielded differently by each approach. Renal and bladder ultrasound (RBUS) is the preferred method for monitoring changes to the upper urinary tract in pediatric patients with neurogenic bladder. ${ }^{16}$ Harbingers of renal damage on RBUS include hydronephrosis, hydroureter, increased bladder wall thickness, and/or impaired bladder emptying. Identification of any of the aforementioned findings may warrant additional imaging studies, such as voiding cystourethrograms (VCUGs), urodynamics, or dimercaptosuccinic acid (DMSA) nuclear renal scans. Guideline recommendations on timing of ultrasound imaging surveillance for upper tract changes are not strictly defined for children with neurogenic bladder. As such, surveillance RBUS imaging intervals vary among treatment centers ${ }^{17}$ and contribute to the diversity of management strategies.

\section{Proactive Approach}

The proactive treatment approach heralds immediate use of CIC with continued use determined by ineffective bladder emptying and/or by findings on early urodynamic (UDS) evaluation. ${ }^{11}$ Surveillance imaging with RBUS is conducted at regular intervals to monitor the upper tracts and bladder for response to therapy or despite it. Furthermore, frequent imaging among patients undergoing proactive treatment can inform potential need for additional testing or intervention. Among centers that implement proactive treatment strategies, RBUS is typically repeated every 3-6 months to assess for upper tract deterioration in infancy, with annual imaging thereafter. ${ }^{17}$ In addition, some entities, such as the European Society for Paediatric Urology, recommend a baseline DMSA scan within the first year of life to detect renal scarring. ${ }^{18}$ The argument for baseline DMSA is that ultrasound has been found to have poor correlation with renal scars and almost half of older patients with neurogenic bladders have renal scarring. ${ }^{19}$

Although prospective studies are currently addressing the question of optimizing imaging intervals, ${ }^{6}$ the need for frequent imaging to detect upper tract and bladder deterioration should be balanced with a realistic follow-up plan that is not overly burdensome to the patient and caregivers. ${ }^{20}$ While frequent imaging can introduce a cost burden to the health system, prevention costs are generally accepted to be less expensive than those for preventable complications or disease progression. $^{21}$ 
Table I Overview of Proactive and Expectant Management Approaches for Spina Bifida

\begin{tabular}{|l|l|l|}
\hline \multirow{2}{*}{} & \multicolumn{1}{|c|}{ Proactive } & \multicolumn{1}{|c|}{ Expectant } \\
\cline { 2 - 3 } & $\begin{array}{l}\text { Early diagnostics and therapeutics prior to laboratory/imaging } \\
\text { evidence of renal dysfunction. }\end{array}$ & $\begin{array}{l}\text { Surveillance labs and imaging prior to proceeding with } \\
\text { invasive diagnostics and therapeutics. }\end{array}$ \\
\hline Imaging & $\begin{array}{l}\text { Frequent renal ultrasound is performed to monitor upper } \\
\text { tracts while patient is on clean intermittent catheterization } \\
\text { (CIC). Routinely performed on surveillance. }\end{array}$ & $\begin{array}{l}\text { Renal ultrasounds are part of the initial assessment and drive } \\
\text { any potential further workup. Routinely performed on } \\
\text { surveillance. }\end{array}$ \\
\hline Labs & $\begin{array}{l}\text { Serum creatinine and cystatin C are regularly measured as } \\
\text { a means to monitor treatment. }\end{array}$ & $\begin{array}{l}\text { Serum creatinine and cystatin C are regularly measured as } \\
\text { a means to potentially prompt further investigation with } \\
\text { more invasive studies. }\end{array}$ \\
\hline $\begin{array}{l}\text { Urodynamics } \\
\text { (UDS) }\end{array}$ & $\begin{array}{l}\text { Encourages the use of UDS within the first few months of life } \\
\text { to obtain baseline information. }\end{array}$ & $\begin{array}{l}\text { UDS is performed only if other studies (imaging or labs) } \\
\text { suggest upper tract deterioration. }\end{array}$ \\
\hline $\begin{array}{l}\text { Catheterization } \\
\text { (CIC) }\end{array}$ & $\begin{array}{l}\text { CIC initiated after birth with continuation determined by } \\
\text { results of UDS or after early UDS demonstrates high risk } \\
\text { parameters. }\end{array}$ & $\begin{array}{l}\text { CIC is not immediately initiated. Patients are followed more } \\
\text { conservatively and the decision to start CIC is dictated by } \\
\text { RBUS, labs, and UDS. }\end{array}$ \\
\hline Pharmacotherapy & $\begin{array}{l}\text { Early initiation of anticholinergics in patients with hostile } \\
\text { bladder pressures on early UDS. }\end{array}$ & $\begin{array}{l}\text { Eot initiated early. Typically initiated if labs and imaging } \\
\text { prompt UDS revealing high risk bladder. }\end{array}$ \\
\hline Surgery & $\begin{array}{l}\text { Eecrease the need for surgical procedures. } \\
\text { Pimilar goal to prevent surgical management with other less- } \\
\text { invasive means. }\end{array}$ \\
\hline
\end{tabular}

\section{Expectant Approach}

Imaging is also a key component of the expectant management approach. In this approach, RBUS imaging is completed as part of the initial assessment and if no hydronephrosis is found, invasive interventions and diagnostics such as CIC, UDS, VCUG, or anticholinergic medications are not initiated. ${ }^{22}$ Of note, VCUG would be performed along with a cystometrogram if UDS is not available. ${ }^{18}$ In the expectant management practice of neurogenic bladder in children with spina bifida, routine RBUS is repeated on a follow-up surveillance schedule. If evidence of upper tract deterioration (eg, new or worsened hydronephrosis) is eventually found on imaging, it serves as the impetus for intervention beginning with UDS and subsequent initiation of CIC, medications, or surgery. ${ }^{22}$

Waiting for changes to be seen in the upper tracts on imaging may delay intervention and negatively impact outcomes. RBUS findings have been shown to be inconsistent with deleterious changes on UDS. ${ }^{23}$ Several centers have reported that a majority of infants with myelomeningocele who underwent UDS immediately after birth had abnormal findings, ${ }^{13,24}$ however, another study reported that in patients under 2 years old, an RBUS is normal in $90 \%$ of the time. ${ }^{25}$ Taken together, these findings suggest that surveillance RBUS may fall short in its ability to identify early urinary tract dysfunction; by the time detrimental changes are seen on RBUS there may have already been irreversible upper tract and/or bladder damage.

Proponents of the expectant management strategy argue that intervening at the time of imaging abnormalities or symptoms is sufficient to protect renal function and avoids early, invasive urologic intervention. ${ }^{22,26}$ In a study of pediatric patients with enuresis, Yeung et al found increased bladder wall thickness on RBUS was correlated with unfavorable parameters on UDS. ${ }^{27}$ Similarly, studies of bladder wall thickness on RBUS in pediatric patients with neurogenic bladders has been shown to correlate with altered UDS parameters, particularly decreased compliance, ${ }^{28}$ suggesting RBUS may be a suitable alternative to more invasive UDS to assess urinary dysfunction. This approach is supported by a study that reported intervention after the onset of RBUS changes resulted in similar renal function and longterm outcomes compared to proactive, prophylactic treatment approaches. ${ }^{26}$ Early urologic interventions potentially could be burdensome to families and premature if outcomes are indeed similar. 


\section{Labs}

Laboratory tests, specifically serum creatinine and cystatin $\mathrm{C}$, are frequently used to monitor patients with $\mathrm{SB}$ but remain controversial in their ability to detect upper urinary tract deterioration. ${ }^{29}$ In patients with normal muscle mass, these values are often used as a proxy for renal function wherein elevated values outside accepted ranges of normal may indicate compromised renal function. Given that many SB patients have low muscle mass, especially in the lower extremities, these tests may be of limited predictive value when compared to other means of assessing upper tract function. ${ }^{17,30}$ Additionally, many standard estimating equations for renal function depend on anthropometric features such as height, which can be difficult to assess in children with SB, scoliosis, and/or joint contractures. ${ }^{29}$ Furthermore, creatinine levels can remain normal with intact unilateral kidney function. ${ }^{16}$ The National Institute for Health and Care Excellence guidelines (UK) for neurogenic lower urinary tract dysfunction specifically recommend against use of isolated serum creatinine or eGFR to assess renal function in this population. $^{30}$

Although recommendations on laboratory tests and testing intervals vary, most guidelines do support the inclusion of biochemical testing as part of routine followup in patients with neurogenic bladder. ${ }^{17}$ Despite this, Chu et al found use of laboratory tests to vary significantly among treatment centers. While $93 \%$ of centers routinely obtained RBUS, only $64 \%$ of centers regularly obtained serum creatinine. ${ }^{17}$ The discordance in testing is not unexpected given the lack of consensus on the specifics of laboratory testing among the guidelines. Whichever laboratory testing strategy is pursued, the decision should be made in context of multidisciplinary care with pediatric nephrology.

\section{Proactive Approach}

In the proactive approach, laboratory measures such as serum creatinine and cystatin $\mathrm{C}$ are regularly measured to assess renal function. Cystatin $\mathrm{C}$ has emerged as a more accurate marker of renal function in individuals with reduced muscle mass, such as children with $\mathrm{SB} .{ }^{31}$ These tests provide a means to monitor for changes in renal function in response to treatment. In children with $\mathrm{SB}$, elevated lab values should prompt evaluation for renal deterioration and subsequent medical or surgical interventions (eg, CIC, anticholinergics, or urinary diversion). ${ }^{26}$

\section{Expectant Approach}

For patients managed expectantly, laboratory values are also considered part of regular surveillance. ${ }^{22}$ As compared to the proactive approach, wherein laboratory values provide a means to monitor change in response to treatment, the expectant approach uses laboratory values, in addition to RBUS and physical exam findings, to prompt further investigation with more invasive studies such as UDS if results are abnormal.

\section{Urodynamics}

Urodynamic evaluation is a critical tool for assessing several aspects of bladder function, including capacity, compliance, storage and leakage pressures, neurogenic detrusor overactivity (NDO), and voiding efficiency. In the neurogenic bladder and SB population, urodynamic findings can identify which patients may be at increased risk for bladder and/ or renal dysfunction, and recurrent UTIs. ${ }^{32,33}$ The preferred approach is video UDS to help detect the presence of reflux and help identify any anatomic abnormalities of the bladder. ${ }^{18}$ Typically, intervention is warranted for detrusor leak-point pressures of $\geq 40 \mathrm{cmH} 2 \mathrm{O}$, consistent with the UMPIRE risk stratification guideline, or findings of NDO and detrusor sphincter dyssynergia (DSD). ${ }^{6,34}$

\section{Proactive Approach}

The proactive approach encourages the early use of UDS has been defined as within the first few months of life for newborns with SB closed at birth. For children with later diagnosis or intervention, it would be ideally performed prior to neurosurgical intervention to obtain baseline information. ${ }^{11}$ Dik et al recommend a proactive approach to neurogenic bladder management, given their findings of lower rates of renal impairment (measured by creatinine clearance) in their SB patient cohort with implementation of periodic UDS, in addition to regular ultrasound and creatinine measurements. ${ }^{16}$ Similarly, Elzeneini et al suggest annual UDS in early childhood, especially if prior studies demonstrated "unsafe" bladder parameters and non-surgical therapies were started or altered. ${ }^{34}$ Given the valuable information regarding bladder parameters provided by UDS, it could be argued that it is important to establish baseline function with UDS early in childhood, ideally shortly after birth, to inform a patient's care trajectory in the short-term. UDS should then be sequentially repeated as the child grows to ensure that no detrimental (but clinically silent) changes are occurring. ${ }^{6}$ 


\section{Expectant Approach}

In this approach, UDS is not routinely performed for baseline or periodic assessment, but rather used when other findings suggest urinary upper tract deterioration. ${ }^{11}$ Periodic, non-invasive assessments with patient history, physical exam, serum creatinine, and RBUS determine need for UDS in the expectant management strategy. If indicated, formal urodynamic evaluation is used to determine bladder function parameters and inform the need for initiating CIC or anticholinergics.

Hopps and Kropp advocated for an expectant approach to use of UDS, finding similar rates of renal impairment in "high risk" patients who received UDS (triggered by ultrasound or exam findings) compared with those that were followed with ultrasound and urine cultures alone. ${ }^{35}$ Similarly, Teichman et al demonstrated equivalent renal deterioration rates among patients with favorable versus impaired results on UDS in SB patients. ${ }^{26,34}$ Thus, they concluded that using UDS is not necessary as a risk stratification tool to determine need for prophylactic CIC or anticholinergic medications.

\section{Interventions}

\section{Clean Intermittent Catheterization (CIC)}

First introduced among patients with spinal cord injuries, use of CIC has expanded to include patients with suspected neurogenic bladder who have bladder emptying dysfunction, particularly SB patients. CIC has been shown to decrease UTIs, decrease renal damage, and improve continence. ${ }^{34,36,37}$ More than $70 \%$ of individuals with SB perform CIC to empty their bladders. ${ }^{38}$ The timing of CIC varies by providers and is somewhat controversial. A 2014 survey of the practice patterns within the British Association of Pediatric Urologists demonstrated an even split between universal catheterization and initiation of catheterization after poor emptying has been established. $^{39}$

\section{Proactive Approach}

The definition of "early" CIC can vary amongst providers and institutions. ${ }^{11}$ In the proactive management approach, patients are placed on scheduled CIC either from birth with continuation determined by results of urodynamic testing or catheterized bladder volumes, ${ }^{11}$ or after early UDS reports high-risk parameters. Early urodynamic assessment may serve to risk-stratify children with the intent to intervene in patients at high risk of progressive bladder dysfunction, defined as poor bladder compliance, high storage pressures (detrusor leak point pressures $>40 \mathrm{~cm}$ of water), or detrusor sphincter dyssynergia. ${ }^{11}$ This proactive method is believed to be better tolerated by families when introduced in infancy and decreases the need for future bladder augmentation, as early management may prevent irreversible bladder neuromuscular and upper urinary tract changes. ${ }^{11,40} \mathrm{CIC}$ is often paired with anticholinergic therapies to decrease detrusor overactivity and/or reduce storage pressures (see pharmacotherapy below). Sometimes, patients may be initiated on overnight catheter drainage which can decrease upper tract dilation and improve continence in children with poorly compliant bladders. ${ }^{41}$ Proponents of early initiation of CIC suggest that the benefits of improved bladder compliance, improved bladder emptying, and conservation of renal function outweigh the risk of early CIC.

Currently, many providers initiate CIC from birth in children with potential for neurogenic bladder and closely monitor post-void residual volumes until results indicate the bladder can adequately empty and the upper urinary tracts do not appear to be at high risk (typically with baseline renal ultrasound with more invasive tests such as VCUG or UDS within the first 3-6 months of life). However, there is significant variability in timing of testing and criteria for risk stratification regarding which patients require continued CIC or other interventions.

A prospective study by $\mathrm{Wu}$ et al examined urologic outcomes in children at high risk for upper tract deterioration on urodynamics (high filling or storage pressures or detrusor sphincter dyssynergia), who were then managed with CIC and anticholinergic medication. ${ }^{40}$ The rate of persistent hydronephrosis was similar in children who were evaluated at less than 1 year (early management) and those evaluated at greater than 4 years of age (expectant management); however, among patients with early initiation of CIC, the rate of bladder augmentation was significantly lower. ${ }^{40}$ Edelstein et al found that patients undergoing CIC demonstrated lower rates of upper tract deterioration, defined as new or worsening hydronephrosis or reflux or increased post-void residual, than those who were simply observed ( 3 of 20 patients and 35 of 40 patients, respectively). ${ }^{42}$ Furthermore, Elzeneini et al found that renal scarring on DMSA scan was less prevalent and occurred later in the clinical course among individuals with SB who practiced proactive CIC compared to a historical cohort that did not practice CIC. ${ }^{34}$ 


\section{Expectant Approach}

In the expectant management strategy, CIC is not immediately initiated. Patients are followed and treated more conservatively, relying on imaging (eg, RBUS and VCUG) instead of baseline UDS testing to inform clinical decision-making. CIC and pharmacotherapy are avoided in patients without adverse upper tract or clinical findings, including normal laboratory measurements of renal function. If abnormalities or complications develop during expectant management, patients are evaluated and started on CIC and/or pharmacotherapy if warranted.

Benefits of expectant management center on reducing invasive interventions, thereby limiting their associated risks and costs. One of the frequently noted goals of the expectant management approach is decreasing family and caregiver anxiety. ${ }^{11,22}$ UDS and CIC both require catheterization, which can be traumatic and can lead to adverse effects such as UTIs, urethral false passage, and hematuria. Additionally, proponents of the conservative approach suggest overtreatment is a concern. Two studies of expectant management have reported $1.2-5 \%$ loss of renal function, suggesting that careful observation with intervention only when necessary or desired for continence may be reasonable. ${ }^{26,35}$ However, these patients were risk-stratified, with highrisk patients (infants with hydronephrosis, urinary retention, febrile UTIs, or vesicoureteral reflux on work-up for infection) undergoing earlier evaluation and intervention. ${ }^{35}$ Low-risk infants were converted to highrisk status if new abnormalities developed on serial observation imaging. Finally, there are concerns that early CIC may predispose patients to UTIs. In a retrospective study by Kaye et al found that infants who were managed with spontaneous voiding had a lower UTI (defined as positive urine culture and associated fever) risk compared to those managed by early CIC. ${ }^{43}$ However, this was a retrospective study and it is possible that patients managed with CIC were high risk and predisposed to UTIs.

Taken together, these studies suggest that proactive CIC may protect upper tract function, reduce upper tract deterioration, and decrease the need for complex bladder augmentation procedures among patients with neurogenic bladder. Expectant use of CIC, while perhaps reducing catheterization-associated risks and anxiety, may permit progression of undesirable outcomes (hydronephrosis, retention, and/or renal damage) related to bladder dysfunction.

\section{Pharmacotherapy}

Anticholinergic pharmacotherapies, such as oxybutynin, solifenacin, and tolterodine, are commonly used in management of symptoms and manifestations of NDO in children and adults with neurogenic bladder dysfunction. Despite their common use in the pediatric patient population, anticholinergic medications for symptoms of NDO, with the exceptions of oxybutynin and solifenacin, are offlabel in children. Importantly, there are notable side effects associated with use of anticholinergic medications in children including behavioral changes, urinary retention, and constipation among others. ${ }^{44}$ Newer drugs such as mirabegron, a beta-3 adrenergic receptor, represent another class of medications that similarly relax the smooth muscle of the urinary bladder and increase bladder capacity. No exact timing or criteria for initiation of anticholinergics exists in current guidelines for SB care or pediatric neurogenic bladder dysfunction generally. As such, use of anticholinergics is largely governed by provider experience or institutional guidelines. ${ }^{5,34}$ Similar to CIC, there is an emphasis on risk-stratifying patients for treatment with anticholinergics based on clinical parameters; however, there is significant variation among centers and among providers in terms of the use of anticholinergics. ${ }^{38}$

\section{Proactive Approach}

The early use of anticholinergics in patients with neurogenic bladder has focused on proactive risk reduction in those with urodynamic evidence of hostile bladder pressures. Early use of anticholinergics in those patients at high risk of bladder dysfunction have several benefits. Primary goals of therapy include decreased detrusor overactivity, reduction in detrusor storage pressures, and reduced incontinence. Additionally, anticholinergics have been shown to affect bladder wall remodeling by decreasing smooth muscle proliferation and can potentially serve as a protection against urinary upper tract deterioration. ${ }^{11,45}$

Lee et al found early initiation of anticholinergics in infants with low bladder capacity or increasing storage pressures had a favorable impact on the bladder on repeat UDS. ${ }^{5}$ These findings were consistent even in patients who did not reach the UMPIRE risk stratification guideline of $>40 \mathrm{cmH} 2 \mathrm{O}$ for high risk detrusor storage pressures. In one study, infants underwent UDS and were started on anticholinergics (21 of 69 patients) at provider discretion (usually if urodynamics demonstrated increased bladder pressure and limited bladder capacity that did not reach 
$50 \%$ of estimated bladder capacity (EBC)). Patients who were started on anticholinergics had a faster rate of bladder growth at 1 year follow-up compared to those who were observed, with the greatest improvement in those with $<50 \%$ EBC. ${ }^{5}$ There was a trend towards improved bladder characteristics in those on anticholinergics. Upper tract deterioration and vesicoureteral reflux were not significantly different between infants taking anticholinergics and those observed. Storage pressures were stable for those on anticholinergics, but increased among patients who were only observed, suggesting that anticholinergics may at least prevent progression of bladder dysfunction. ${ }^{5}$

These results support the initiation of anticholinergics for any increase in bladder storage pressures in infants. This may be applicable to older children as well; however, bladder function and parameters change with natural growth in infants and children without intervention, so ascribing benefits is complicated.

\section{Expectant Approach}

A more expectant management encourages conservative use of anticholinergic pharmacotherapy. Pharmacotherapy is rarely initiated during early bladder development because UDS is not performed early in this group. Rather, pharmacotherapy is typically initiated when laboratory data and/or imaging prompts UDS evaluation or when a child/family desires continence. The decision to initiate pharmacotherapy can also be influenced by reports of voiding symptoms from the child, parents, or care providers. Proponents of the expectant approach suggest that the longevity and maintenance of improvement in bladder parameters remain unclear, ${ }^{5}$ such that early management decisions may not have the long-lasting effects that are initially seen in some studies. Additionally, anticholinergic side effects are not insignificant and in a study from Lee et al, $25 \%$ of patients experienced side effects of oxybutynin administration. ${ }^{5}$ The long-term effects of improving lower urinary tract parameters with anticholinergic pharmacotherapy on urinary upper tract function are currently not well-known.

\section{Surgery}

When medical management interventions fail or are not completely effective, surgical interventions may be necessary to protect renal function or achieve social continence. Among these options is chemodenervation of the bladder via intra-detrusor onabotulinumtoxinA injections, which are used primarily in cases of treatment-resistant NDO or severe incontinence. ${ }^{46}$ Published clinical response rates to chemodenervation injections in pediatric patients vary from $53 \%$ to $100 \% .{ }^{47}$ Urinary diversions, such as vesicostomy or ileal conduit, are examples of incontinent surgical procedures for neurogenic bladder dysfunction that may or may not be temporary. More complex bladder reconstruction, such as enterocystoplasty, is performed to lower bladder storage pressures, increase bladder capacity, and/or attain continence. ${ }^{48}$

\section{Proactive Approach}

Enterocystoplasty is a complex operation that carries significant short- and long-term complication rates, although modern institutional series have shown decreased morbidity than earlier studies. ${ }^{2,10,49}$ As such, early emphasis on non-surgical interventions, including CIC and anticholinergic medications, has been pursued in an attempt to decrease the need for procedures with higher morbidity and complications. ${ }^{42,48}$

Bladder reconstruction rates vary widely among institutions and regions. ${ }^{2}$ Further, outcome measures are not uniform across institutions, so assessing surgical success in achieving the intended goals is difficult. Reassuringly, continence rates and satisfaction are high, despite relatively high complication rates. ${ }^{48}$ Notably, bladder augmentation (typically ileocystoplasty) is performed more often than urinary diversion (typically with ileal conduit). ${ }^{49}$

\section{Expectant Management}

Wang et al found a significant trend toward decreasing bladder reconstruction rates and increasing chronic renal insufficiency rates in the SB population. ${ }^{10}$ While these data were drawn from an administrative database (National Inpatient Sample), the study noted that the trend toward more conservative therapy for SB patients may lead to worse renal protection, though direct correlations are difficult to reliably make from non-longitudinal data sources.

\section{Outcomes}

\section{Urinary Tract Infections}

UTIs can accelerate the renal damage caused by high pressure reflux or elevated bladder storage pressures, thus timely intervention to maintain low bladder pressures is crucial to preventing renal deterioration. ${ }^{13}$ The diagnosis of UTIs in this population are especially difficult given the high rate of catheterization and some patients with bowel segments in their bladder. Colonization of urine is very 
common and diagnosis of UTI relies on both culture data and symptoms. ${ }^{50}$ Proponents of early use of CIC would argue that proper bladder management reduces the increased risk of UTI with bladder instrumentation, but the risk. In the study from Hopps and Kropp, for example, of the 65 infants in the expectant management group, the most common reason for conversion to a high-risk and initiation of CIC was a febrile UTI $(\mathrm{n}=29,45 \%) .{ }^{35}$ However, in a retrospective cohort study of patients with $\mathrm{SB}$, participants who spontaneously voided had lower risk of UTI compared to those using CIC. ${ }^{43}$ As noted above in the CIC section, this study was retrospective and did not provide risk stratification of participants. It is possible that those in the spontaneous voiding group had low risk bladders which was the reason why they were less likely to have UTIs.

\section{Continence}

Achieving continence, if desired by the patient/parent, is one of the primary goals of spina bifida management. A number of studies have explored continence as an outcome when comparing proactive and expectant management approaches. Wu et al compared initiation of CIC at 1 year of age to 4 years of age and found similar continence rates between the two groups. ${ }^{40}$ Kaefer et al compared an expectant group (CIC and anticholinergic initiated only when upper tract disease manifested) and a proactive group (CIC and anticholinergic initiated if baseline UDS was concerning). Incontinence rates were similar in the two groups, with 6 of 27 patient incontinent in the expectant group and 3 of 18 incontinent in the proactive group. Overall, continence rates did not appear to differ between the two treatment approaches.

\section{Hydronephrosis/Upper Tract Deterioration}

For patients with bladder dysfunction, there is consensus that hydronephrosis is reduced when interventions such as CIC are performed. One study found that early identification of detrusor sphincter dyssynergia on UDS with subsequent implementation of CIC led to a significant reduction in hydroureteronephrosis on follow-up. ${ }^{16}$ At onset of the study, five renal units demonstrated slight dilation and four units demonstrated gross dilation. In the follow-up period, ten renal units demonstrated slight dilation and one unit demonstrated severe dilation. ${ }^{16}$ Another study found a greater number of patients with hydronephrosis in their expectant management group, even with or without vesicoureteral reflux, compared with the prophylactic treatment group. $^{23}$

\section{Renal Failure/Scarring}

Renal injury and chronic kidney disease (CKD) are the result of unmanaged bladder dysfunction. Protection against renal dysfunction is paramount, as progression to CKD or dialysis can impart worsened morbidity and mortality outcomes. Early intervention for bladder dysfunction takes a proactive approach to protection of renal function. Dik et al reported that of 144 patients (286 functioning kidneys) undergoing proactive treatment, unilateral parenchymal scars were suspected in 10 patients with only 6 kidneys having confirmed parenchymal damage on DMSA scan (2.1\% of kidneys) during the follow-up period, three of which were notably cases wherein patients were untreated prior to 6 months of age. ${ }^{16}$ Furthermore, serum creatinine was initially normal in all patients; on follow-up, 2 of 103 patients had creatinine clearance $<80 \mathrm{~mL} / \mathrm{min}$ (one attributed to short stature and one to unilateral grade 5 VUR with reflux nephropathy). ${ }^{16}$ However, the data for renal function preservation does not clearly favor one method. Hopps and Kropp found that renal function deterioration occurred in only $1.2 \%$ of their study population, even if upper tract deterioration was also present. ${ }^{35}$ Importantly, 44\% of their initial "low risk" study population (29 of 65 patients) progressed to "high risk" (febrile UTI, new onset hydronephrosis, urinary retention, or vesicoureteral reflux) during the follow-up period, a significant increase over the mean follow-up of 10 years. $^{35}$ The authors' preference focuses on slowing progression of renal dysfunction and reducing preventable complications, such as pyelonephritis and retention, by utilizing early intervention.

\section{Conclusion}

Management of pediatric neurogenic bladder dysfunction in patients with SB is widely variable in clinical practice and in the literature. In absence of a standardized, guideline-directed approach, both proactive and expectant management strategies exist. The proactive management strategy is characterized by early urodynamic evaluation, CIC, and anticholinergic pharmacotherapy, to detect high-risk detrusor pressures 
and intervene before renal dysfunction progresses. The expectant management approach utilizes surveillance laboratory and ultrasound imaging without invasive evaluation or interventions, a conservative approach that is safe. While both methods are regularly employed with literature to supporting both sides, early intervention strategies such as those described in the CDC-funded UMPIRE trial seem have a greater benefit in preventative management and, in modern studies, may decrease risk of renal dysfunction compared with expectant management. Nevertheless, rigorous prospective research and systematic review of the available data is needed to further determine the optimal treatment strategies for children with spina bifida.

\section{Abbreviations}

SB, spina bifida; UTI, urinary tract infection; CIC, clean intermittent catheterization; UDS, urodynamic study; RBUS, renal and bladder ultrasound; DMSA, dimercaptosuccinic acid; VCUG, voiding cystourethrogram; EBC, estimated bladder capacity; CDC, Centers for Disease Control.

\section{Author Contributions}

Dr. Hobbs and Ms. Krischak conceptualized and designed the study, collected and analyzed data, drafted the initial manuscript, and reviewed and revised the manuscript.

Dr. Tejwani collected, analyzed data, and reviewed and revised the manuscript.

Dr. Routh conceptualized and designed the study, coordinated and supervised data collection, and reviewed and revised the manuscript.

Drs. Wiener and Purves conceptualized and designed the study and critically reviewed the manuscript for important intellectual content.

All authors contributed to data analysis, drafting or revising the article, gave final approval of the version to be published, agreed to the submitted journal, and agree to be accountable for all aspects of the work.

\section{Funding}

This article was not funded by an outside sponsor.

\section{Disclosure}

Dr Jonathan C Routh is a Consultant for Spina bifida association, outside the submitted work. The authors have no relevant financial and non-financial relationships to the article to disclose.

\section{References}

1. Ouyang L, Bolen J, Valdez R, Joseph D, Baum MA, Thibadeau J. Characteristics and survival of patients with end stage renal disease and spina bifida in the United States renal data system. J Urol. 2015;193(2):558-564. doi:10.1016/j.juro.2014.08.092

2. Routh JC, Joseph DB, Liu T, et al. Bladder Reconstruction Rates Differ among Centers Participating in National Spina Bifida Patient Registry. $J \quad$ Urol. 2018;199(1):268-273. doi:10.1016/j. juro.2017.08.084

3. Lloyd JC, Wiener JS, Gargollo PC, Inman BA, Ross SS, Routh JC. Contemporary epidemiological trends in complex congenital genitourinary anomalies. J Urol. 2013;190(4 Suppl):1590-1595. doi:10.1016/j.juro.2013.04.034

4. Parker SE, Mai CT, Canfield MA, et al. Updated National Birth Prevalence estimates for selected birth defects in the United States, 2004-2006. Birth Defects Res A Clin Mol Teratol. 2010;88 (12):1008-1016. doi:10.1002/bdra.20735

5. Lee AS, Viseshsindh W, Long CJ, et al. How early is early? Effect of oxybutynin on bladder dynamics within the first year of life in patients with spina bifida. J Pediatr Urol. 2020;16(2):168 e1-168 e6. doi:10.1016/j.jpurol.2019.12.008

6. Routh JC, Cheng EY, Austin JC, et al. Design and Methodological Considerations of the Centers for Disease Control and Prevention Urologic and Renal Protocol for the Newborn and Young Child with Spina Bifida. J Urol. 2016;196(6):1728-1734. doi:10.1016/j. juro.2016.07.081

7. Dudley AG, Adams MC, Brock JW 3rd, et al. Interrater Reliability in Interpretation of Neuropathic Pediatric Urodynamic Tracings: an Expanded Multicenter Study. J Urol. 2018;199(5):1337-1343. doi:10.1016/j.juro.2017.12.051

8. Schechter MS, Liu T, Soe M, Swanson M, Ward E, Thibadeau J. Sociodemographic attributes and spina bifida outcomes. Pediatrics. 2015;135(4):e957-64. doi:10.1542/peds.2014-2576

9. Joseph DB, Baillie S, Baum MA, Frimberger DC, Khavari R, Misseri R, Tanaka ST, Wood H, Yerkes EB. Urology. Guidelines for the Care of People with Spina Bifida, 4th edition. 2018;162-175.. Avaibale from: https://www.spinabifidaassociation.org/resource/guidelinespd full/. Accessed August 23, 2021.

10. Wang HH, Lloyd JC, Wiener JS, Routh JC. Nationwide Trends and Variations in Urological Surgical Interventions and Renal Outcome in Patients with Spina Bifida. J Urol. 2016;195(4 Pt 2):1189-1194. doi:10.1016/j.juro.2015.11.033

11. Edwards AB, Jacobs M. Early Vs. Expectant Management of Spina Bifida Patients-Are We All Talking About a Risk Stratified Approach? Curr Urol Rep. 2019;20(11):76. doi:10.1007/s11934-019-0943-Z

12. Snow-Lisy DC, Yerkes EB, Cheng EY. Update on Urological Management of Spina Bifida from Prenatal Diagnosis to Adulthood. J Urol. 2015;194(2):288-296. doi:10.1016/j.juro.2015.03.107

13. Bauer SB, Hallett M, Khoshbin S, et al. Predictive value of urodynamic evaluation in newborns with myelodysplasia. JAMA. 1984;252 (5):650-652. doi:10.1001/jama.1984.03350050038023

14. Szymanski KM, Misseri R, Whittam B, Kaefer M, Rink RC, Cain MP. Quantity, Not Frequency, Predicts Bother with Urinary Incontinence and its Impact on Quality of Life in Adults with Spina Bifida. J Urol. 2016;195 (4 Pt 2):1263-1269. doi:10.1016/j.juro.2015.07.108

15. Inouye BM, Jiang R, Alkazemi MH, et al. Hospital and ED charges for spina bifida care in the United States between 2006 and 2014: over \$2 billion annually. Disabil Health J. 2019;12(3):431-436. doi:10.1016/j.dhjo.2019.01.007

16. Dik P, Klijn AJ, van Gool JD. Early Start to Therapy Preserves Kidney Function in Spina Bifida Patients. Eur Urol. 2006;49 (5):908-913. doi:10.1016/j.eururo.2005.12.056

17. Chu DI, Liu T, Patel P, et al. Kidney Function Surveillance in the National Spina Bifida Patient Registry: a Retrospective Cohort Study. J Urol. 2020;204(3):578-586. doi:10.1097/JU.0000000000001010 
18. Stein R, Bogaert G, Dogan HS, et al. EAU/ESPU guidelines on the management of neurogenic bladder in children and adolescent part I diagnostics and conservative treatment. Neurourol Urodyn. 2020;39 (1):45-57. doi:10.1002/nau.24211

19. Veenboer PW, Hobbelink MG, Ruud Bosch JL, et al. Diagnostic accuracy of Tc-99m DMSA scintigraphy and renal ultrasonography for detecting renal scarring and relative function in patients with spinal dysraphism. Neurourol Urodyn. 2015;34(6):513-518. doi:10.1002/nau.22608

20. de Kort LM, Bower WF, Swithinbank LV, Marschall-Kehrel D, de Jong TP, Bauer SB. The management of adolescents with neurogenic urinary tract and bowel dysfunction. Neurourol Urodyn. 2012;31 (7):1170-1174. doi:10.1002/nau.22206

21. Gray JAM. Redefining Health Care: creating Value-Based Competition on Results. BMJ. 2006;333(7571):760. doi:10.1136/ bmj.333.7571.760

22. Frimberger D, Cheng E, Kropp BP. The current management of the neurogenic bladder in children with spina bifida. Pediatr Clin North Am. 2012;59(4):757-767. doi:10.1016/j.pcl.2012.05.006

23. Kaefer M, Pabby A, Kelly M, Darbey M, Bauer SB. Improved bladder function after prophylactic treatment of the high risk neurogenic bladder in newborns with myelomentingocele. $J$ Urol. 1999;162(3 Pt 2):1068-1071. doi:10.1097/00005392-19990900000031

24. Parizi JLG, Leal da Cruz M, Andrade MC, et al. A Comparative Analysis of Bladder Pattern of Patients who Underwent In Utero Versus Postnatal Myelomeningocele Repair. J Urol. 2020;203 (1):194-199. doi:10.1097/JU.0000000000000521

25. Kessler TM, Lackner J, Kiss G, Rehder P, Madersbacher H. Early proactive management improves upper urinary tract function and reduces the need for surgery in patients with myelomeningocele. Neurourol Urodyn. 2006;25(7):758-762. doi:10.1002/nau.20304

26. Teichman JM, Scherz HC, Kim KD, Cho DH, Packer MG, Kaplan GW. An alternative approach to myelodysplasia management: aggressive observation and prompt intervention. J Urol. 1994;152(2 Pt 2):807-811. doi:10.1016/s0022-5347(17)32716-7

27. Yeung CK, Sreedhar B, Leung VT, Metreweli C. Ultrasound bladder measurements in patients with primary nocturnal enuresis: a urodynamic and treatment outcome correlation. J Urol. 2004;171 (6 Pt 2):2589-2594. doi:10.1097/01.ju.0000112978.54300.03

28. Şekerci ÇA, Işbilen B, Işman F, Akbal C, Şimşek F, Tarcan T. Urinary NGF, TGF- $\beta 1$, TIMP-2 and bladder wall thickness predict neurourological findings in children with myelodysplasia. $J$ Urol. 2014;191(1):199-205. doi:10.1016/j.juro.2013.08.025

29. Szymanski KM, Szymanski AJ, Salama AK, Hains DS, Cain MP, Misseri R. Estimating and tracking renal function in children and adults with spina bifida. J Pediatr Urol. 2020;16(2):169-177. doi:10.1016/j.jpurol.2019.12.009

30. National Clinical Guideline C. National Institute for Health and Clinical Excellence: guidance. Urinary Incontinence in Neurological Disease: management of Lower Urinary Tract Dysfunction in Neurological Disease. Royal College of Physicians (UK) Copyright (C) 2012, National Clinical Guideline Centre; 2012.

31. Pham-Huy A, Leonard M, Lepage N, Halton J, Filler G. Measuring glomerular filtration rate with cystatin $\mathrm{C}$ and beta-trace protein in children with spina bifida. $J$ Urol. 2003;169(6):2312-2315. doi:10.1097/01.ju.0000060205.23406.13

32. Yeung CK, Sreedhar B, Leung YF, Sit KY. Correlation between ultrasonographic bladder measurements and urodynamic findings in children with recurrent urinary tract infection. BJU Int. 2007;99 (3):651-655. doi:10.1111/j.1464-410X.2006.06580.x

33. Prakash R, Puri A, Anand R, Jain AK, Lal B, Garg V. Predictors of upper tract damage in pediatric neurogenic bladder. J Pediatr Urol. 2017;13(5):503.e1-503.e7. doi:10.1016/j.jpurol.2017.02.026
34. Elzeneini W, Waly R, Marshall D, Bailie A. Early start of clean intermittent catheterization versus expectant management in children with spina bifida. J Pediatr Surg. 2019;54(2):322-325. doi:10.1016/j. jpedsurg.2018.10.096

35. Hopps CV, Kropp KA. Preservation of renal function in children with myelomeningocele managed with basic newborn evaluation and close followup. $J$ Urol. 2003;169(1):305-308. doi:10.1097/01. ju.0000040590.35948.bc

36. Lapides J, Diokno AC, Silber SJ, Lowe BS. Clean, intermittent self-catheterization in the treatment of urinary tract disease. $J$ Urol. 1972;107(3):458-461. doi:10.1016/s0022-5347(17)61055-3

37. Verpoorten C, Buyse GM. The neurogenic bladder: medical treatment. Article. Pediatr Nephrol. 2008;23:717. doi:10.1007/s00467007-0691-z

38. Liu T, Ouyang L, Thibadeau J, et al. Longitudinal Study of Bladder Continence in Patients with Spina Bifida in the National Spina Bifida Patient Registry. J Urol. 2018;199(3):837-843. doi:10.1016/j. juro.2017.11.048

39. Lee B, Featherstone N, Nagappan P, McCarthy L, O'Toole S. British Association of Paediatric Urologists consensus statement on the management of the neuropathic bladder. J Pediatr Urol. 2016;12 (2):76-87. doi:10.1016/j.jpurol.2016.01.002

40. Wu HY, Baskin LS, Kogan BA. Neurogenic bladder dysfunction due to myelomeningocele: neonatal versus childhood treatment. J Urol. 1997;157(6):2295-2297. doi:10.1016/S0022-5347(01)64766-9

41. Nguyen M, Pavlock C, Zderic S, Carr M, Canning D. Overnight catheter drainage in children with poorly compliant bladders improves post-obstructive diuresis and urinary incontinence. J Urol. 2005;174:1633-6; discussion $1636 . \quad$ doi:10.1097/01. ju.0000179394.57859.9d

42. Edelstein RA, Bauer SB, Kelly MD, et al. The long-term urological response of neonates with myelodysplasia treated proactively with intermittent catheterization and anticholinergic therapy. $J$ Urol. 1995;154(4):1500-1504. doi:10.1016/S0022-5347(01)66914-3

43. Kaye IY, Payan M, Vemulakonda VM. Association between clean intermittent catheterization and urinary tract infection in infants and toddlers with spina bifida. J Pediatr Urol. 2016;12(5):284.e1-284.e6. doi:10.1016/j.jpurol.2016.02.010

44. Reid SM, Westbury C, Guzys AT, Reddihough DS. Anticholinergic medications for reducing drooling in children with developmental disability. Dev Med Child Neurol. 2020;62(3):346-353. doi:10.1111/ dmen. 14350

45. Çetinel B, Önal B, Can G, Talat Z, Erhan B, Gündüz B. Risk factors predicting upper urinary tract deterioration in patients with spinal cord injury: a retrospective study. Neurourol Urodyn. 2017;36 (3):653-658. doi:10.1002/nau.22984

46. Gamé X, Mouracade P, Chartier-Kastler E, et al. Botulinum toxin-A $\left(\right.$ Botox ${ }^{\circledR}$ ) intradetrusor injections in children with neurogenic detrusor overactivity/neurogenic overactive bladder: a systematic literature review. J Pediatr Urol. 2009;5(3):156-164. doi:10.1016/j.jpurol.2009.01.005

47. Kask M, Rintala R, Taskinen S. Effect of onabotulinumtoxinA treatment on symptoms and urodynamic findings in pediatric neurogenic bladder. $J$ Pediatr Urol. 2014;10(2):280-283. doi:10.1016/j. jpurol.2013.09.004

48. Scales CD Jr, Wiener JS. Evaluating outcomes of enterocystoplasty in patients with spina bifida: a review of the literature. $J$ Urol. 2008;180(6):2323-2329. doi:10.1016/j.juro.2008.08.050

49. Wiener JS, Antonelli J, Shea AM, et al. Bladder augmentation versus urinary diversion in patients with spina bifida in the United States. J Urol. 2011;186(1):161-165. doi:10.1016/j.juro.2011.03.023

50. Tradewell M, Pariser JJ, Nimeh T, Elliott SP. Neurogenic Bladder Research G. Systematic review and practice policy statements on urinary tract infection prevention in adults with spina bifida. Transl Androl Urol. 2018;7(Suppl 2):S205-S219. doi:10.21037/ tau.2018.04.21 


\section{Publish your work in this journal}

Research and Reports in Urology is an international, peer-reviewed, open access journal publishing original research, reports, editorials, reviews and commentaries on all aspects of adult and pediatric urology in the clinic and laboratory including the following topics: Pathology, pathophysiology of urological disease; Investigation and treatment of urological disease; Pharmacology of drugs used for the treatment of urological disease. The manuscript management system is completely online and includes a very quick and fair peer-review system, which is all easy to use. Visit http://www.dovepress.com/ testimonials.php to read real quotes from published authors.

Submit your manuscript here: https://www.dovepress.com/research-and-reports-in-urology-journal 